\title{
A Discussion on the Application of Internet of Things Technology in Intelligent Agriculture
}

\author{
Jing Li \\ Xi’an Peihua University, Xi’an, Shaanxi, 710125, China
}

Keywords: Internet of Things; Intelligent Agriculture; Informationization; Framework

\begin{abstract}
With the application of the Internet of Things and other new technologies, the transformation of traditional agriculture has been continuously improved. As a representative of modern agriculture, intelligent agriculture has occupied a dominant position in agricultural development. To build intelligent agriculture, it is necessary to profoundly understand the connotation of the Internet of Things and rely on it to build automatic agriculture. This will help enhance the intelligence of agricultural production, fully realize its informationization and accelerate science and technology achievements transformation. Based on the author's learning and practical experience, this paper first analyzed the concept of agricultural Internet of Things and its application in intelligent agriculture. Then the author proposed the framework of intelligent agricultural Internet of Things. Finally, this paper discussed the application of Internet of Things in intelligent agriculture.
\end{abstract}

\section{Introduction}

The rapid development of the Internet industry has changed people's traditional way of life, broken the limitations of traditional industries and played a pivotal role in agriculture and industry. It promotes the integration of urbanization and agricultural modernization. At present, "Internet +" has been applied in all walks of life. In agriculture, intelligent agricultural application based on the Internet of Things, such as intelligent agricultural irrigation, greenhouses and fisheries, has been gradually developed. Actively adopting Internet of Things technology in agriculture and actively advancing agricultural informationization and modernization in China, has become an inevitable direction for future agricultural development and an inevitable choice for developing intelligent agriculture.

\section{The Concept of Agricultural Internet of Things and Its Application in Intelligent Agriculture}

\subsection{The concept of agricultural Internet of Things}

The concept of the Internet of Things was first proposed by Professor Ashton of the Massachusetts Institute of Technology in 1999. The Internet of Things is a network connecting objects based on Internet. The Internet of Things is widely used in network convergence by virtue of intelligent perception technology, recognition technology, universal technology and other communication-aware devices. This is also known as the third wave of information industry development after computers and the Internet. Agricultural Internet of Things refers to the application of Internet of Things technology in agriculture. It applies a large number of temperature, humidity, image acquisition and RFID sensors to all aspects of agricultural production, such as aquaculture, animal husbandry, greenhouse cultivation and facility gardening. In other aspects, with the monitoring network which is composed of network equipment, servers and application software, a relatively uniform data transmission and heterogeneous data conversion protocol can be established, so that animal and plant life, production tools, environmental elements and other physical components in the agricultural system can be connected with various virtual parts on the Internet. With the agricultural Internet of Things, intelligent identification, location, tracking, 
monitoring and management of agricultural objects can be realized. The closed-loop control of the agricultural environment can be realized with intelligent control terminals, thus achieving high informationization, automation and intelligence of agricultural production.

\subsection{The application of Internet of Things in intelligent agriculture}

Intelligent agriculture refers to linking Internet o Things technology with traditional agriculture based on sensors and software, and with certain platforms to regulate agricultural production and make agriculture smarter. In this process, personalized management of differences in seeds, fertilizers and pesticides caused by differences in soil composition, fertilization needs and crop growth cycles can be achieved through the Internet of Things technology. Moreover this can automatically record the production process of crops to ensure the quality of agricultural products, thus providing traceable information to regulators and consumers. In the current situation, it is generally difficult for traditional agriculture to achieve these functions and intelligence agriculture can effectively solve these problems. The specific measures are as follows:

(1) Monitoring function: it can rely on wireless sensors to realize real-time supervision of farmland and collect data on temperature, humidity and light intensity. Farmers can use video to monitor the growth of crops and remotely control farmland environment. In addition, with the Internet of Things technology, the collected data and the obtained information can be converted into digital data which can be transmitted to the agricultural intelligent management system by the network. The system can control various types of equipment to realize the intelligent management of crop growth process according to various elements in the growth of the crops. (2) Supervising function: With the RFID electronic tag, the agricultural product safety traceability system can be constructed to realize information sharing and transparent management of crop production, transportation and sales, and to ensure its safety. This will promote the creation of crop brands and increase the added value of its products. (3) Real-time image and video surveillance function: Using the Internet of Things to manage agriculture is to rely on the network to establish the connection between crops, cultivated land and soil. Based on multi-dimensional information and with the multi-level processing methods, the growth environment of crops can be controlled. With the Internet of Things system, a unified data mode can be established by connecting and comparing agricultural production data and historical environmental data in different conditions. Through data mining and other technologies, the system can analyze environmental parameters that can improve assist decision-making in promoting crop growth and crop yield.

\section{The Framework of Intelligent Agricultural Internet of Things}

\subsection{System structure design}

The intelligent agricultural Internet of Things system includes information collectors, intelligent gateways, intelligent monitoring equipment and automatic control facilities. The principle of its composition is that the information collector is used to collect environment-related content, convert its signal into an analog signal and then convert it into a digital signal by means of an analog-to-digital converter. Then it will access the wireless transmission facility to convert the signal into electromagnetic waves. The wireless transmission facility uses zigbee technology, which not only consumes less energy, but also has a large space for network construction. Therefore, it enables multi-terminal access and completes data transmission relying on the coordinator of the facility. The intelligent gateway implements comprehensive processing of information and transmits signals to the supervisory center. The monitoring center realizes the analysis and preservation of information. At the same time, the expert system intelligently judges the control command to transmit it to the automatic controller to control the devices according to the collected information. On the other hand, the live images are gathered into the monitoring center for remote visual monitoring, and experts can give suggestions. The system framework is shown in Figure 1. 

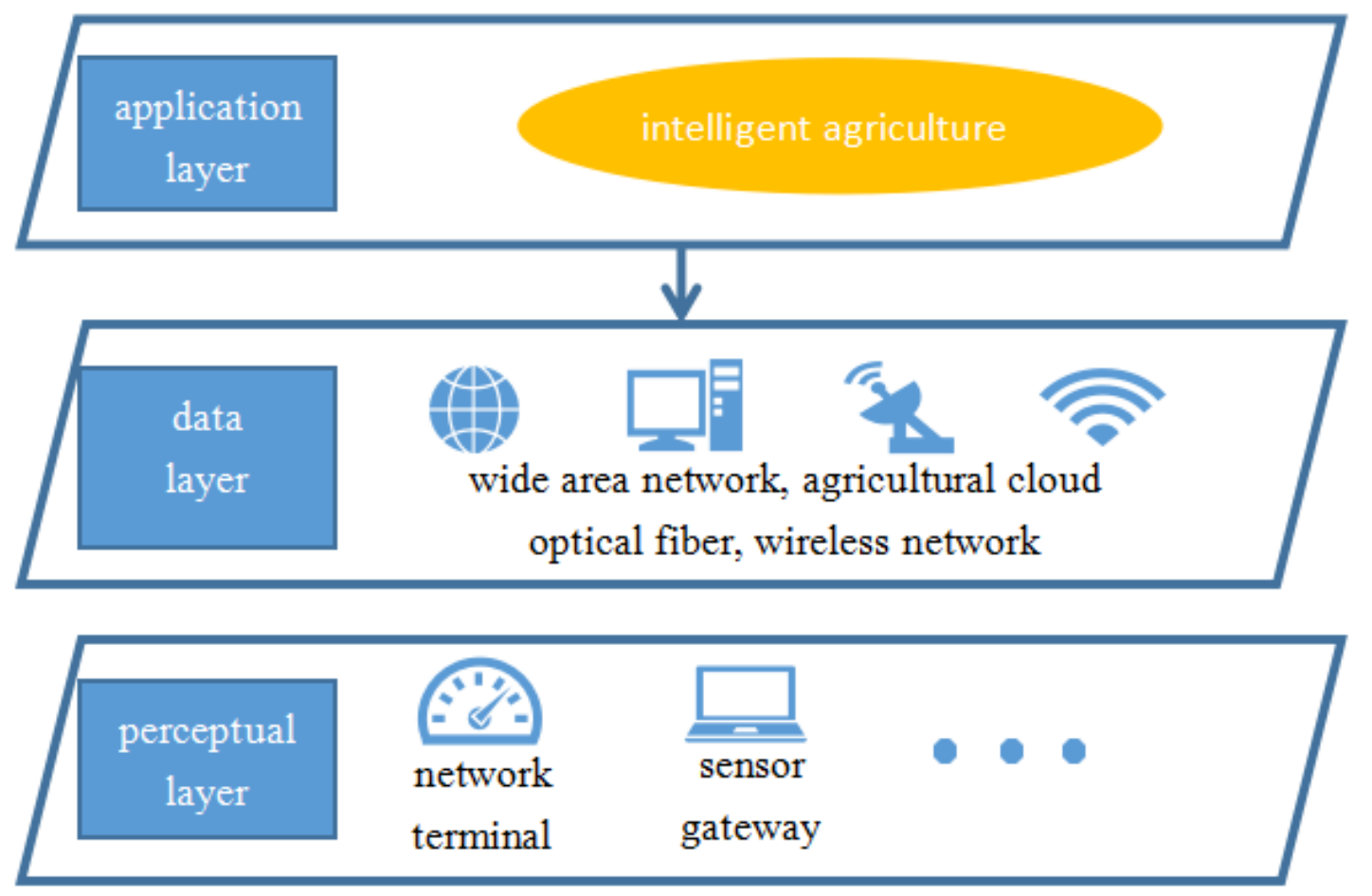

Fig. 1 Composition of Internet of Things in intelligent agriculture

\subsection{The perceptual layer of Internet of Things}

The perceptual layer of Internet of Things mainly includes sensors, RFID devices and video monitoring devices. Their main function is data collection. The corresponding data collected by the zigbee node, CAN node and other communication modules is transmitted to the intelligent gateway on the Internet of Things to complete real-time testing and collection of on-site data. The application system is at the upper level. The instructions it conveys can be transmitted to the relay based on the intelligent gateway on the Internet of Things. This can help regulate the switch of the agricultural equipment and complete the adjustment of the agricultural production environment.

\subsection{The network layer of Internet of Things}

The network layer of Internet of Things relies on the integration of LAN, WLAN, CDMA and 4G networks to achieve real-time and accurate transmission and exchange of farmland data information and control commands. The perceived agricultural production information is transmitted to the required places in an accessible, fast, high-security and reliable manner, thus enabling the goods to be communicated over long distances and on a large scale.

\subsection{The application layer of Internet of Things}

The application layer of Internet of Things is the highest level of intelligent agriculture and is users-oriented. It can build different operating platforms according to user needs. It is mainly used to realize real-time access and data sharing of information about agricultural production and circulation of agricultural products, so as to ensure correct pre-production planning to improve resource utilization efficiency, precise production ,efficiency and circulation. This can achieve safety traceability and increase sales channels to promote high yield, quality, efficiency, ecology and safety of agriculture. The application layer of Internet of Things contains multiple application systems to identify agricultural production environment, control process and prevent disease. At the same time, it can also analyze and process the information obtained by the perceptual layer of Internet of Things to complete the regulation, management and decision making of agricultural production. 


\section{The Application of Internet of Things in Intelligent Agriculture}

\subsection{Intelligent agricultural production based on the Internet of Things}

By virtue of various sensors and corresponding transmission equipment, the field information can be immediately transmitted to relevant management personnel. This establishes the connection between farmers and farmland. With the help of label technology, it is also possible to construct a modern logistics storage base to accelerate the delivery rate and achieve food supervision throughout the process to ensure safety. The obtained farmland information and agricultural materials will be used to monitor natural disasters. Early warning will be carried out. This can facilitate regional management, so that information can be shared in an all-round way and the degree of agricultural automation can be improved.

\subsection{Intelligent agricultural management based on the Internet of Things}

Internet of Things technology can significantly improve the management level of traditional agriculture. In agricultural production, agricultural intelligent sensors and networks can be used to collect and disseminate agricultural environmental data and provide a basis for the control of farmland environment. This can improve the agricultural production environment. It not only brings a good production environment for crops and increases production, but also saves investment in materials, such as fertilizers.

\subsection{Intelligent supervision of agricultural products based on the Internet of Things}

In the production and circulation of agricultural products, comprehensive use of electronic labels, bar codes and other equipment can complete the quality supervision and traceability of crops and food with the support of mobile communications and computer network systems. This can achieve long-distance visual management. In this way, real-time supervision and intelligent control of the whole process of crops in the field, crop processing and food sales can be realized. Digital logistics can be achieved in crops and food, which not only improves the speed of transportation, but also directly improves the quality of food.

\section{Summary}

As the third wave of the development of the information industry, the Internet of Things has attracted the attention of various countries. China is a big agricultural country, and the development of the Internet of Things can be used as a new innovation point and economic growth driver. Its development challenges us and provides opportunities. Through the analysis of the traditional Internet of Things, this paper improved the structure of the existing agricultural Internet of Things system, analyzed the problems in each layer and then introduced the application of the Internet of Things. However, the agricultural Internet of Things is a very large system engineering, people need to make breakthroughs in hardware, software, network, management and policy. The development of agricultural Internet of Things has great potential and it is developing towards industrialization and informatization. The application of agricultural Internet of Things technology plays s a positive role in promoting sustainable development, advancing the scientific management and production operations of green agriculture and improving the production process of crops. Intelligent agriculture will gradually replace traditional agricultural production methods. With the support of national policies, the investment of fund projects and the application of of cloud computing, big data, cloud services and artificial intelligence, people can establish agricultural expert systems and knowledge decision-making models. The development of Internet of Things equipment suitable for different production environments plays an important role in transforming China's traditional agriculture and developing modern agriculture. 


\section{References}

[1] Zhan Yong. The Application of Intelligent Agriculture Technology in Vegetable Greenhouse Design [J]. Construction Materials and Decoration, 2018, (40): 97-98.

[2] Li Feng. Intelligent Agriculture Promotes Industrial Transformation and Upgrading [J]. China Agricultural Reclamation, 2018, (09): 45-47.

[3] Hou Zhongxin, Liu Chuntao and Lu Na. A Discussion on the Construction of Qingdao Intelligent Agricultural Meteorological Service [J]. Agriculture and Technology, 2018, 38(14): 242.

[4] Chen Si, Ding Wei and Chen Siheng. An Applied Research of Internet of Things in Intelligent Agriculture [J]. Southern Agricultural Machinery, 2018, 49 (14): 120. 